Rev. High Pressure Sci. Technol., Vol. 7 (1998) 1514 1516

\title{
Mechanism of Plastic Flow of Gaskets and Anvils in 6-8 Type Anvils
}

\author{
J. Yoshimoto \\ National Institute for Research in Inorganic Materials, Tsukuba, Ibaraki 305, Japan
}

\begin{abstract}
In the 6-8 type anvils, the occurrences of blow-out have restricted the attainable pressure to $25 \mathrm{GPa}$. The cause of blow-out was attributed to the fracture of the cube of the second stage anvils made of cemented tungsten carbide (WC). The stress state in the cube was, therefore, calculated by a finite element method. As a result, there was a fracture line in the stress diagram of $\sigma_{0}$ vs $\sigma_{\mathrm{m}}$ that was expressed as $\sigma_{0}=\mathrm{S}_{0}-(3 / 2) \sigma_{\mathrm{m}}$, where $\sigma_{0}, \sigma_{\mathrm{m}}$, and $\mathrm{S}_{0}$ were the equivalent stress of von Mises, the mean normal stress, and half of the compressible strength, respectively. Furthermore, the breakage of pyrophyllite was also proven to follow the same fracture line except for the value of $\mathrm{S}_{0}$. In Bridgman, anvils the expression of failure stress which derived from our flow model on the basis of the fracture line, accounted for the compressible strength of the various thicknesses of gaskets. The observed plastic deformation of the cube of the second stage anvils would already occur at lower pressures when the $\sigma_{0}$ exceeded the parallel fracture line, where the $S_{0}$ was less than the value at failure.
\end{abstract}

[6-8 type anvils, pressure generation, yield criterion, gasket flow, plastic deformation]

\section{Introduction}

A 6-8 type anvil is a double-stage pressure generating system studied first by Kawai and Endo [1]. It functions as a high-temperature apparatus with a considerable volume octahedral pressure chamber in the pressure range of $20 \mathrm{GPa}$ and is markedly available for scientists who wants to find jewels in the bosom of the earth. We have been studying the pressure generation of the 6-8 type anvils for 21 years. However, the attainable pressure has also been $25 \mathrm{GPa}$ for 21 years, using the WC cube with a truncation size of 2 $\mathrm{mm}$ as second stage anvils and both the octahedral pressure medium and the gasket made of pyrophyllite of which the starting size was $5.8 \mathrm{~mm}$ in the side length of octahedron and $1.5 \mathrm{~mm}$ in both thickness and width of the form of wing, respectively, because the blow-out always occurs in the neighborhood of the load corresponding to the highest possible pressure. The stress induced in the gaskets had been already measured; as a result, the gaskets were more stable as the pressure rose, while the stress difference increased with the pressure. Moreover, the broken cube was always one of the eight second stage anvils and in addition, a tip of it covered with the gaskets sank in. Consequently, these experimental results explained that the origin of the blowout was not attributed to the gaskets' instability but the failure of the anvil [2].

The attainment of the generation of pressure which is higher than $25 \mathrm{GPa}$, would be supposed to be beyond the strength capability of the cube made of WC as the second stage anvils. It is usually accepted that the choice of harder substances as anvils were an important factor in approaching higher pressure, and large-size sintered diamonds are then produced these days by high pressure-technology. Hence, cubes made of sintered diamond instead of WC, enabled the realization of a new generation of pressure in the range of 30 $\mathrm{GPa}$. Getting the hardest possible materials, such as sintered diamonds for the anvils, we could reach higher pressures without any considerations [3]. The mechanical strength of sintered diamond is higher than that of WC certainly, but not so significantly, now, at the most, two times higher. The nominal engineering strength would be gradually increased by some improvements of the sintering process, but the highest possible strength should be finite. Therefore, the direct way which depends only on the effect of the strength of the anvil material, is enormously available for pressure generation, but sets an upper limit to the attainable pressure. On the other hand, if the stress difference at any point in an anvil did not exceed the mechanical strength of an anvil, an arbitrary high pressure could be produced by using a material with finite strength, without failure of the anvils [4]. However, it is not shown how such sophisticated stress distribution is realized in the anvil and gasket system. It does not propose a powerful technique to attain an arbitrary high pressure by actual apparatus. We have measured many factors to analyze 6-8 type anvils under loading. At this times, we can not suggest a powerful way to produce an arbitrary high pressure, but we can analyze the failure of the anvil, the flow of the gaskets and the yielding of the anvil.

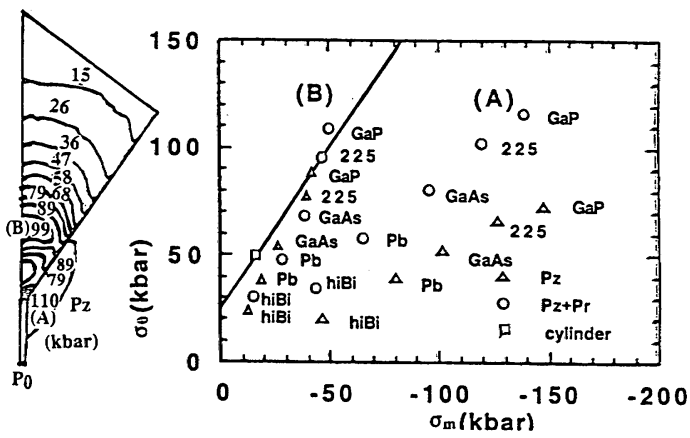

Fig. 1. Contour lines of equivalent stress of von Mises at the pressure of $25 \mathrm{GPa}$. (the left side)

Fig. 2. The plot of two maximums of $\sigma_{0}$ against $\sigma_{\mathrm{m}}$ at every pressure of calibration. (the right side)

\section{Mechanism of Failure of an Anvil}

The loading distributions on the faces of a cube and on the surface of the truncated corner, generated stress fields within the body of a cube anvil, which were calculated approximately as an axisymmetric body by the finite element method. The stress distributions in the gaskets were obtained by the measurement of the thickness of the gasket as a function of distance from the truncated edge corner, and in the octahedron, the pressure medium were assumed to be uniform pressure. Then, the contour lines of equivalent stress of von Mises $\left(\sigma_{0}\right)$, at the pressure of $25 \mathrm{GPa}$ [5] that corresponds to the metal-insulator transition point of gallium phosphide, is shown in Fig. 1 as a typical result of calculated stress fields. The shape of contour map is characterized by each component of stress and is identical except for the values of the lines at every pressure of 
calibration. There are two points of nearly equal maximum $\sigma_{0}$ in the anvil as shown in Fig. 1. One of them is near by the face of truncated corner (A) and another is in the body along the central axis at the equivalent position of the periphery of the gasket on the surface of the anvil (B). Both maximums exceeded the critical value at the pressure of 18 $\mathrm{GPa}$ that corresponded to the uniaxial stress for failure of the cylindrical rod. The anvils, however, did not break at this pressure nor did blow-out occur. Is the $\sigma_{0}$ of no use as a criterion for failure?

The difference of two maximums of $\sigma_{0}$ was clarified by considering a mean normal stress $\left(\sigma_{\mathrm{m}}\right)$ of another component of stress in addition to the $\sigma_{0}$. All the maximums of $\sigma_{0}$ calculated at every pressure of the calibration were plotted against $\sigma_{\mathrm{m}}$ at the same position, as shown in Fig. 2. The compressive strength of WC is also shown in Fig. 2 and this point is connected to the point of $\mathrm{GaP}$ of (B), because only compressive strength and $\mathrm{GaP}$ points in the points of Fig. 2 correspond to the state of failure. Besides, the extrapolation of this line was found to intersect the ordinate at a point half of the compressive strength. This line was, therefore, defined as a fracture line that divided the stress diagram of $\sigma_{0}$ vs. $\sigma_{\mathrm{m}}$ into two areas, failure and success. It was expressed as follows:

$$
\sigma_{0}=S_{0}-(3 / 2) \sigma_{m}
$$

where

$$
\mathrm{S}_{0} \text { : half of compressive strength. }
$$

The appearance of breakage of the cube was a depression at the tip of it covered with gaskets. It was in fair agreement with the yielding of area (B) derived from Eq. (1).

Generally, $\sigma_{\mathrm{m}}$ and $\sigma_{0}$ are an invariant of the first and second order according to orthogonal transformation of coordinates, respectively; they are not tensor but scalar. If we consider the shear stress instead of $\sigma_{0}$, Eq. (1) would be identical to an equation of the maximum shear stress principle that is modified by the assumption of the internal friction according to the normal stress in place of $\sigma_{m}$. On the other hand, Eq. (1) is not a tensor equation, but rather a scalar. A scalar represents a quantity of state, and therefore, is reasonable to be criterion for failure. In fact, the equivalent stress of von Mises $\sigma_{0}$ corresponds to the uniaxial stress component of stress states, and von Mises yield criterion is, hence, the stress for fracture of cylindrical rod. The von Mises yield criterion is based on the $\sigma_{0}$ alone. Certainly, pressure is of no effect for failure, but may be effective for criterion. Accordingly, Eq. (1) is considered to be a higher order approximation than the von Mises criterion.

Furthermore, the destruction tests had been made on the tapered anvils of WC to investigate the effect of the massive support principle by Tsujii in the past [6]. The experimental results have been calculated by the finite element method, and as a result, the failure of the tapered anvils obeyed Eq. (1) as well as cube 6-8 type anvils, for the maximum of $\sigma_{0}$ of them was located on the fracture line, as shown in Fig. 3. Therefore, the fracture line of Eq. (1) explained two facts with no relation at first and elucidated the massive support principle. In addition, we made tapered anvils of pyrophyllite instead of WC, and the destruction tests were also made on them as the previous part of the gasket flow study. They also conformed to Eq. (1), and of course the value of $\mathrm{S}_{0}$ was different from that of WC. In addition, sintered diamond also followed Eq. (1) [7]. Finally, the coefficient of $\sigma_{\mathrm{m}}$ had $3 / 2$ in common with the materials mentioned-above. If $\mathrm{S}_{0}$, which is characterized by the absence of $\sigma_{\mathrm{m}}$, is half of the compressive strength of the material like the maximum shear stress criterion, it should always be $3 / 2$ in the three-dimensional stress state.

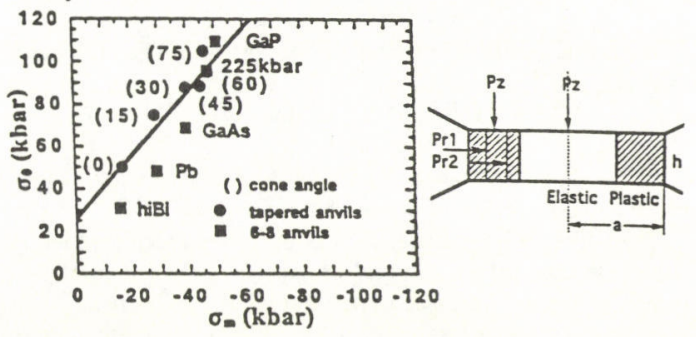

Fig. 3. The plot of maximums of $\sigma_{0}$ against $\sigma_{\mathrm{m}}$ for tapered anvils and 6-8 type anvils. (the left side)

Fig. 4. Balance of force in the flowing part of gasket

\section{Gasket Flow arising from the Failure}

In Bridgman anvils, high pressure is generated by extruding a proper thin gasket within opposed anvils. If the thickness of the gasket is unusually increased before loading, the compression of it would be identical to the destruction test for determining the compressive strength of its material. Pyrophyllite of unusual thickness was compressed within opposed anvils while measuring the advancement of the anvil. The strength of it was determined by the value of the load of accelerative advancement of the anvil. Although it has inherent quantity of material, the results of our measurements depended on the thickness, from the points of mean pressure in Fig. 5. When we carefully observed the advancement of the anvil, however, it was the out of linear advancement at the load that corresponded to the strength of pyrophyllite. Hence, at any thickness, all the gasket parts reach uniform failure point at the pressure of $2 \mathrm{~S}_{0}$, and the periphery of the gasket starts to flow out along the radial direction on the flat face of the anvils. The flow of the gasket is then assumed to be stopped due to the friction between the gasket and anvils, and the radial component of stress distribution is consequently induced within the gasket in addition to axial stress. The radial component of stress, always 0 in the periphery of the gasket, increases as compression towards the center and therefore, the stress state in the inner part of the gasket, recovers to the elastic state from the plastic state according to Eq. (1). An increment of the load would be applied on the elastic part, only axial stress increases, and the stress state again reaches the failure point. The previous process is repeats and then flowing part of the gasket goes on slowly towards the center [8]. The area of the elastic part shrinks and then, the load is concentrated on the central part of the gasket.

The balance of force in the flowing part of the gasket is retained as follows:

$$
h\left(P_{r}^{1}-P_{r}^{2}\right) \leq 2 \mu P_{z} \Delta r
$$

where

$\mathrm{P}_{\mathrm{r}}$ : radial component of stress

$\mathrm{P}_{\mathrm{Z}}$ : axial component of stress

$\Delta r$ : distance of radial direction between two adjacent points and

$$
\mu \text { : coefficient of friction }
$$

as shown in Fig. 4. If maximum slope of radial stress distribution was realized in Eq. (2), the equal sign would be valid. Moreover, when $\Delta \mathrm{r}$ limits to infinitesimally small, Eq. (2) becomes

$$
\mathrm{dP}_{\mathbf{r}} / \mathrm{dr}=(2 \mu / \mathrm{h}) \mathrm{P}_{\mathbf{Z}}
$$


As discussed in the previous section, the destruction of pyrophyllite obeyed the failure criterion of Eq. (1). Then $P_{r}$ and $\mathrm{P}_{\mathrm{Z}}$ satisfy the relation such that

$$
\mathrm{P}_{\mathrm{z}}=4 \mathrm{P}_{\mathrm{r}}+2 \mathrm{~S}_{0} \text {. }
$$

Integration of Eq. (3) under the condition of Eq. (4) now yields

$$
\begin{array}{lll}
\text { or } & \mathrm{P}_{\mathrm{r}}(\mathrm{r})=(1 / 2) \mathrm{S}_{0}(\exp ((4 \mu / \mathrm{h})(\mathrm{a}-\mathrm{r}))-1) \\
& \mathrm{P}_{\mathrm{Z}}(\mathrm{r})=2 \mathrm{~S}_{0} \exp ((4 \mu / \mathrm{h})(\mathrm{a}-\mathrm{r}))
\end{array}
$$

where $a$ is the radius of center flat of anvil.

The values of $\mu$ at every data point, were calculated as to coincide the surface integral of Eq. (6) on the face of the anvil with the values of experimental load. Then Eq. (6), which has a proper value of $\mu$ by adjustment, explains the relation between the strength and the thickness of gaskets, as shown in Fig. 5. Therefore, Bridgman anvils are identical to the destruction test of a cylindrical rod and only the inner elastic part is available for a gasket. Nothing causes the extrusion of a gasket except a uniaxial load according to the failure condition of Eq. (1). Higher axial stress can be produced by a thinner gasket on the whole, and critical thickness of the gasket that corresponds to shear strength by Wakatsuki is not an inherent quantity of material [9]. This is because the critical thickness of pyrophyllite gasket was different between WC and a sintered diamond anvil [7]. The destruction line of Eq. (1) is, therefore, essential for consideration of gasket flow $[10,11]$.

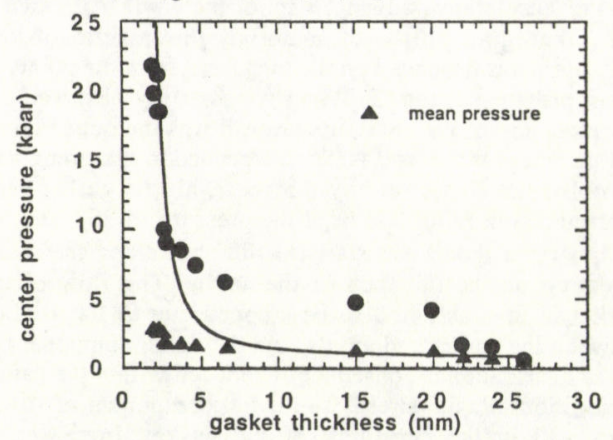

Fig. 5. Strength of pyrophyllite as a function of thickness.

\section{Plastic Deformation of Cube Anvils}

Fig. 6 shows the plastic deformation of cube 6-8 type anvils after unload. It is clear that plastic deformation has already occurred at the pressure of $12 \mathrm{GPa}$. Generally speaking, to determine whether the maximum of $\sigma_{0}$ is beyond the critical value or not, the von Mises yield criterion is sufficient. The $\sigma_{0}$ that exceeds Eq. (1) at first,

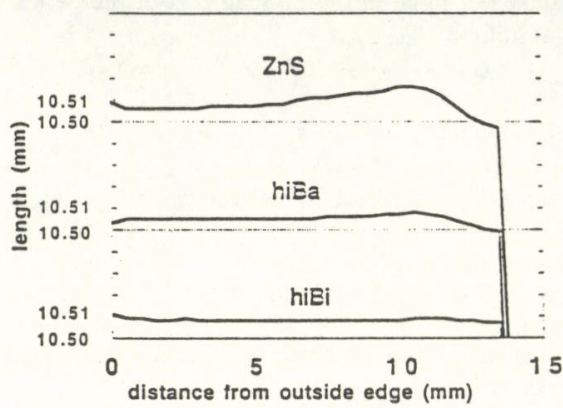

Fig. 6. Plastic deformation of cube after unload. however, may not be always the maximum of $\sigma_{0}$ in the case of the criterion of Eq. (1). Hence, all $\sigma_{0}$ of cube anvil at the pressure of $12 \mathrm{GPa}$ is plotted against $\sigma_{\mathrm{m}}$ as shown in Fig. 7. No $\sigma_{0}$ exceeds the fracture line of Eq. (1), where $S_{0}$ is $2.5 \mathrm{GPa}$. The cube nevertheless suffered plastic deformation. The $\mathrm{S}_{0}$ of $2.5 \mathrm{GPa}$ is obtained from a destruction test of WC. The $S_{0}$ occurring at the initial plastic deformation, would be consequently supposed to be a smaller value, with estimates of approximately $1.5 \mathrm{GPa}$ from Fig. 7 at the pressure of $12 \mathrm{GPa}$. In addition, the maximum of $\sigma_{0}$ did not obviously exceed the fracture line at first in all the $\sigma_{0}$. When all the points in Fig. 6 are assumed to follow Eq. (1), the values of tentative $S_{0}$ of them can be calculated by the substitution of $\sigma_{0}$ and $\sigma_{\mathrm{m}}$ into Eq.(1). The largest tentative $\mathrm{S}_{0}$ is considered first of all to exceed the fracture line and then, the tentative $S_{0}$ evaluates directly the plastic deformation. Hence, the contour map of the tentative $S_{0}$ at the pressure of $12 \mathrm{GPa}$ supposes that the tip of the anvil, covered by the gaskets, is going to depress along the fault with the form of a cone, as shown in Fig. 8. At this point, this is considered to coincide roughly with the measurement of the plastic deformation as shown in Fig. 6, and we emphasize finally, that the tip part of the anvil was free from plastic deformation until failure occurred.
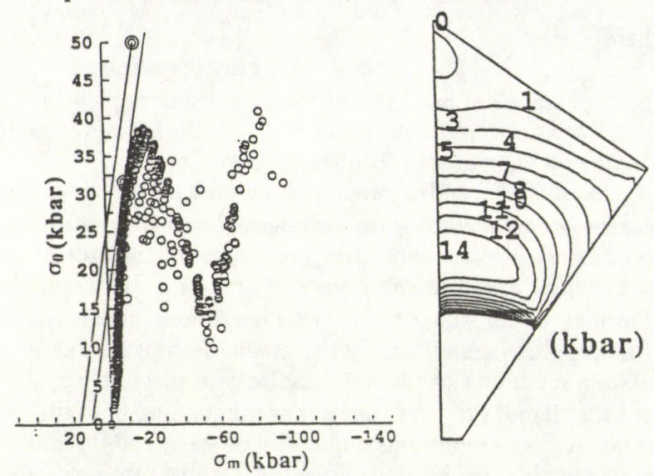

Fig. 7. The plot of all $\sigma_{0}$ in the cube against $\sigma_{\mathrm{m}}$ at $12 \mathrm{GPa}$. (the left side)

Fig. 8. Contour lines of tentative $\mathrm{S}_{0}$ at $12 \mathrm{GPa}$.

\section{References}

[1] N.Kawai and S.Endo, Rev. Sci. Instrumm.. 41, 1178 (1970)

[2] J.Yoshimoto, Proc. 26th. High. Pressure. Cof. Jpn. 202 (in Japanese) (1993)

[3] F.P.Bundy, Rev. Sci. Instrum., 48, 591 (1977)

[4] M.Kumazawa, High Temp - High Press., 5, 599(1973)

[5] A.Ohtani and A.Onodera, Atsuryoku Gizyutsu, 18, 225(in Japanese) (1980)

[6] S. Tsujii and M.Jinushi, Proc. 11th. High Pressure. Cof. Jpn. 15 (in Japanese) (1970)

[7] T. Taniguchi, O.Shimomura, and S.Yamaoka, Jpn. J. Appl. Phys. , 33, 4012 (1993)

[8] J.Yoshimoto, Proc. 34th. High.Pressure. Cof. Jpn. 181 (in Japanese) (1993)

[9] M. Wakatsuki, K.Ichinose, and T.Aoki, Jpn. J. Appl. Phys., 11, 578 (1972)

[10] B. Okai and J.Yoshimoto, Jpn. J. Appl. Phys., 10, 534(1971)

[11] B. Okai and J.Yoshimoto, High Temp - High Press., 5, 675 (1973) 